\title{
CÁNULAS NASALES DE ALTO FLUJO. ¿APORTAN VALOR?
}

Artículo original: Ni Y-N, Luo J, Yu H, Liu D, Ni Z, Cheng J, et al. Can High-flow Nasal Cannula Reduce the Rate of Endotracheal Intubation in Adult Patients With Acute Respiratory Failure Compared With Conventional Oxygen Therapy and Noninvasive Positive Pressure Ventilation? Chest. 2017;151(4):764-75.

\section{Autor de correspondencia: Federico Gordo Vidal}

\section{Resumen Estructurado:}

Antedecentes: Existe controversia sobre los efectos de las Cánulas Nasales de alto Flujo (CNAF) en el tratamiento de la insuficiencia respiratoria aguda (IRA) en pacientes adultos. El objetivo del estudio es establecer si el empleo de CNAF puede reducir la tasa de reintubación, en este grupo de pacientes, en comparación con la oxigenoterapia convencional (OC) o el empleo de ventilación no invasiva (VNI).

Método: Búsqueda bibliográfica en las principales bases de datos y registros de ensayos clínicos, así como información de Sciences Institute Web of Science, de todos los ensayos clínicos comparando el empleo de CNAF con $\mathrm{OC}$ y/o VNI en pacientes adultos con IRA. El desenlace principal estudiado fue la tasa de intubación endotraqueal y los secundarios fueron la mortalidad y el tiempo de estancia en UCI.

Resultado: Incluyen en el metanálisis 18 ensayos clínicos con 3.881 pacientes. En estos estudios no se encontró heterogeneidad en sus resultados salvo para el análsis de la mortalidad en UCI (I2 67\%, Chi2 12.21, P 0.02) y la tasa de intubación endotraqueal (I2 63\%, Chi2 13.51, P 0.02 ) entre CNAF y VNI. El empleo de CNAF se asoció con una menor tasa de intubación en comparación con $\mathrm{OC}$ ( $\mathrm{P}$ 0.01), mientras que no hubo diferencia en comparación con la VNI (P 0.16). El empleo de CNAF no se asoció a reducción en la mortalidad ni estancia en UCI.

Conclusión: En pacientes adultos con IRA, el empleo de CNAF es una alternativa sencialla al empleo de la $\mathrm{VNI}$ y reduce la tasa de intubación endotraqueal en comparación con la OC.

\section{Comentario:}

La IRA es una complicación frecuente, de múltiples entidades clínicas, que 
supone una causa muy frecuente de ingreso en UCI y tiene una morbimortalidad elevada. Un número muy importante de estos pacientes requieren tratamientos que incluyen el empleo de ventilación mecánica (invasiva o no invasiva) en $\mathrm{UCl}$ y esto lo convierte en un proceso realmente relevante en la práctica clínica en continua evaluación. Tanto la ventilación invasiva como la VNI son procesos no exentos de complicaciones y por tanto se buscan alternativas que eviten que los pacientes tengan que ser sometidos a estos procedimientos.

Por otro lado, a día de hoy conocemos que los pacientes con IRA en ventilación espontánea que presentan un aumento del trabajo respiratorio, también tienen riesgo de empeorar su lesión pulmonar debido al incremento en la presión transpulmonar que se puede producir también, y de forma inadvertida en ventilación espontánea (1). Este hecho, posiblemente va a cambiar en breve las indicaciones del soporte ventilatorio y la monitorización de los pacientes en ventilación espontánea con el objetivo de una mejor valoración del trabajo respiratorio y no solo del intercambio y equilibrio gaseoso.

El empleo de CNAF es una técnica de soporte de la oxigenación, y posiblemente de la ventilación, que ha emergido recientemente y ha irrumpido en la práctica clínica en los servicios de Medicina Intensiva y también en servicios de Urgencias hospitalarias. Esta técnica se basa en el empleo de dispositivos que son capaces de administrar altos flujos de oxígeno, que pueden llegar a sobrepasar los 100 litros por minuto, mediante un sistéma de adaptación a la nariz del paciente y acondicionamiento del aire suministrándole una temperatura adecuada y consiguiendo humidificar este gas de modo que mejora tanto la posibilidad de intercambio gaseoso como la tolerancia por parte de los pacientes a este flujo de gas (2).

El mecanismos de funcionamiento de las CNAF en la IRA no está plenamente aclarado de modo que este efecto no parece depender únicamente de la cantidad de oxígeno suministrada al paciente, sino que se ha descrito que este sistema puede disminuir el espacio muerto (mejorarndo por tanto la eficiencia de la ventilación) y puede producir un efecto modesto de CPAP que puede incrementar la capacidad residual funcional (abriendo zonas de posible colapso pulmonar) y puede mejorar las condiciones mecánicas de la ventilación. Esto hace que incluso se esté expandiendo su utilización a pacientes no solo hipoxémicos sino también hipercápnicos (3).

Sin embargo, en el momento actual no existe unanimidad en cuanto a la verdadera eficacia y eficiencia en diferentes indicaciones del empleo de CNAF. Ni y col. (4) realizan esta revisión sistemática con el objetivo de 
establecer, en pacientes adultos con IRA, si el empleo de CNAF se asocia a mejora en el pronóstico. El desenlace principal objetivo del estudio es la reducción en la tasa de intubación, mientras que emplean como desenlaces secundarios otros como el tiempo de estancia o la mortalidad en UCI. En la revisión sistemática no analizan otros factores que pueden tener relación con la utilización de la técnica como pueden ser las complicaciones asociadas con el uso de CNAF o VNI y los episodios de intolerancia con ambas técnicas. Estas circunstancias a mi juicio pueden interferir y explicar en parte los resultados obtenidos tanto en los ensayos clínicos como en la práctica real.

Realizan una búsqueda de todos los ensayos clínicos registrados en las bases de datos convencionales y hacen también una busqueda iterativa en la bibliografía de los artículos publicados entre 1996 y 2016. Identifican 776 artículo incluyendo finalmente en el metanálisis 18 que recogen la intubación o no en sus resultados. De los 18 estudios incluidos, 12 fueron ensayos clínicos siendo el resto análisis retrospectivos o prospectivos de cohortes. Estos estudios incluyen 3.881 pacientes de los que 1.893 fueron tratados con CNAF, 934 con VNI y 1.054 con OC.

Llama la atención en este metanálisis la selección de artículos de series de casos (cuando hay 11 ensayos clínicos disponibles) y también llama mucho la atención la inclusión de algunos artículos que realmente no analizan el efecto sobre la IRA sino el efecto sobre la prevención de la misma postextubación. Estos dos hechos sin duda pueden artefactar los resultados del presente metanálisis como se demuestra por la gran heterogeneidad que se refiere en los diferentes resultados de los estudios. Además no hubo retirada de artículos del análisis por criterios metodológicos, cuando varios estudios tenían una gran posibilidad de bias debido a su diseño (no ciego).

El principal resultado del metanálisis es que el tratamiento con CNAF en comparación con OC reduce la tasa de intubación (OR 0.47 [95\% IC, 0,27 a $0,84]$ ), mientras que no reduce la tasa de intubación en comparación con VNI (OR 0.73 [95\% IC, 0,43 a 1,13)]. No encontraron diferencias significativa ni en el tiempo de estancia en $\mathrm{UCl}$ ni en la mortalidad con respecto al empleo de CNAF vs OC o VNI.

Es muy interesante un análsis posthoc que realizan en un subgrupo muy importante de pacientes en los que el motivo es el empleo postextubación. En este subgrupo de pacientes el empleo de CNAF reduce la necesidad de reintubación frente al empleo de OC, pero no frente al empleo de VNI y no hay cambio tampoco en el resto de desenlaces. 
Realmente los resultados del presente estudio son decepcionantes y no parece aportar mayor nivel de evidencia del que aportan los ensayos clínicos incluidos en el mismo. En el momento actual se están extendiendo las indicaciones de uso de CNAF fundamentalmente por su facilidad de empleo en la práctica clínica, sin embargo deberíamos exigir un mayor nivel de evidencia para su utilización y la realización de análsisis de coste efectividad.

En este sentido es de gran interés el análisis publicado por MayordomoColunga y col. (5) que analizan el coste que puede suponer cada intervención en una patología concreta en el que plantea que a la luz del posible coste económico y con los datos actuales, en el caso de las crisis asmáticos en niños no este justificado el empleo de sistemas de CNAF. Los autores plantean diferente escenarios en función de la posibilidad de la superioridad o no del empleo de CNAF y realizando un estudio de costes nos muestran como en el caso de evitar una reintubación aplicando a la población que describen tendría un coste incremental de 1.734 dolares (95\%Cl: 991 a 4.955 .2$)$.

En el momento actual quizás podamos contemplar un escenario de empleo estratificado de los diferentes sistemas de oxigenoterapia convencional, CNAF, VNI y ventilación invasiva. Estas técnicas deben utilizarse con una indicación adecuada y con monitorización estricta que nos permita reducir las complicaciones y evitar un retraso en la intubación que conocemos puede dar lugar a un peor pronóstico. Sin duda las CNAF tienen su papel, pero tenemos que hacer un esfuerzo por tener estudios en diferentes indicaciones, con adecuada calidad metodológica y que incluyan un análisis económico real de lo que supone su utilización.

Con lo que conocemos ahora y empleando un análisis de tipo Bayesiano y aplicando un escenario de referencia (sin entusiasmo, ni escepticismo), podríamos decir que hay un $2,8 \%$ de probabilidad de que la VNI sea superior a CNAF, un $97 \%$ de probabilidad de que sean equivalentes y solo un $0 \%$ de probabilidad de que el empleo de CNAF sea superior a la VNI (5). A día de hoy aún estamos teniendo estudios que demuestran resultados de equivalencia entre las diferentes estrategias (6).

A día de hoy CNAF son una alternativa más en el tratamiento de la IRA en pacientes adultos, pero que en ningún caso debe retrasar un empleo adecuado de la Ventilación Mecánica. A la hora de elegir la técnica a emplear, sobre todo entre CNAF y VNI debemos tener en cuentan tanto factores dependientes del paciente, como de su patología, como de la experiencia y disponibilidad de la técnica. No existe evidencia suficiente 
como para justificar una sustitución de la VNI por sistemas de CNAF y deberíamos tener muy en cuenta el posible coste incremental que supone la disponibilidad de estos equipos.

Dr. Federico Gordo Vidal

Servicio de Medicina Intensiva. Hospital Universitario del Henares. (Coslada-Madrid) Universidad Francisco de Vitoria.

EMAIL DE CONTACTO: fgordo5@gmail.com

\section{REFERENCIAS}

1. Yoshida T, Roldan R, Beraldo MA, Torsani V, Gomes S, De Santis RR, et al. Spontaneous Effort During Mechanical Ventilation: Maximal Injury With Less Positive End-Expiratory Pressure. Crit Care MeD. 2016;44(8):e678-88.

2. Masclans JR, Pérez-Terán P, Roca O. Papel de la oxigenoterapia de alto flujo en la insuficiencia respiratoria aguda. Med Intensiva. 2015 Nov;39(8):505-15.

3. Plotnikow G, Thille AW, Vasquez D, Pratto R, Desmery P. High-flow nasal cannula oxygen for reverting severe acute exacerbation of chronic obstructive pulmonary disease: A case report. Med Intensiva 2017. Ahead of print. DOI: 10.1016/j.medin.2016.11.009

4. Ni Y-N, Luo J, Yu H, Liu D, Ni Z, Cheng J, et al. Can High-flow Nasal Cannula Reduce the Rate of Endotracheal Intubation in Adult Patients With Acute Respiratory Failure Compared With Conventional Oxygen Therapy and Noninvasive Positive Pressure Ventilation? Chest. 2017;151(4):764-75.

5. Mayordomo-Colunga J, Medina A. High-flow nasal cannula oxygenation for everyone? Not so fast! Med Intensiva. 2017. Ahead of print.

DOI:10.1016/j.medin.2017.03.003

6. Fernandez R, Subira C, Frutos-Vivar F, Rialp G, Laborda C, Masclans JR, et al. High-flow nasal cannula to prevent postextubation respiratory failure in highrisk non-hypercapnic patients: a randomized multicenter trial. Ann Intensive Care. 2017;7(1):47 